\title{
Nicotine dependence and functional connectivity of insular cortex subregions
}

Dara G. Ghahremani, Ph.D. ${ }^{1 *}$, Jean-Baptiste Pochon, Ph.D. ${ }^{1 *}$, Maylen Perez Diaz, Ph.D. ${ }^{1}$,

Rachel F. Tyndale, Ph.D. ${ }^{2,3}$, Andy C. Dean, Ph.D. ${ }^{1,5}$, and Edythe D. London, Ph.D. ${ }^{1,4,5}$

${ }^{1}$ Department of Psychiatry and Biobehavioral Sciences, Semel Institute for Neuroscience and Human Behavior, University of California, Los Angeles, CA, USA.

${ }^{2}$ Department of Pharmacology \& Toxicology and Department of Psychiatry, University of Toronto, 1 King's College Circle, Toronto, ON, Canada.

${ }^{3}$ Campbell Family Mental Health Research Institute, Centre for Addiction \& Mental Health, Toronto, ON, Canada

${ }^{4}$ Department of Molecular and Medical Pharmacology, and ${ }^{5}$ Brain Research Institute, University of California, Los Angeles, CA, USA.

* Contributed equally to this work

Corresponding Authors:

Dara Ghahremani, Ph.D. \& Edythe D. London, Ph.D.

Semel Institute for Neuroscience and Human Behavior

760 Westwood Plaza, Room C8-831

Los Angeles, CA 90095-1759

Phone: (310) 825-0606

Fax: (310) 825-0812

E-mail: darag@ucla.edu, elondon@mednet.ucla.edu

Number of figures and tables: 3 figures, 2 tables

Supplemental material: 13 tables 


\begin{abstract}
The insular cortex has been identified as a promising target in brain-based therapies for Tobacco Use Disorder, and has three major sub-regions (ventral anterior, dorsal anterior, and posterior) that serve distinct functional networks. How these subregions and associated networks contribute to nicotine dependence has not been well understood, and therefore was the subject of this study. Forty-seven individuals (24 women; 18-45 years old) who smoked cigarettes daily rated their dependence using the Fagerström Test for Nicotine Dependence (FTND), abstained from smoking overnight $(\sim 12 \mathrm{~h})$, and underwent resting-state functional MRI. Correlations between dependence and resting-state functional connectivity (RSFC) of the major insular subregions were evaluated using whole-brain-corrected voxel-wise analyses and post-hoc region-ofinterest (ROI) analyses. Dependence was analyzed both as a unitary (FTND total score) and bivariate construct - two FTND factors ("morning smoking" and "daytime smoking"). Dependence was negatively correlated with connectivity of both the right dorsal and left ventral anterior insula with the left precuneus, and with connectivity of the left posterior insula to the left putamen. In post-hoc analyses, dependence correlated negatively with connectivity between all anterior insula subregions and the left precuneus, and with bilateral posterior insula connectivity with the left posterior putamen. The latter finding was driven by "daytime smoking". These results suggest an anterior-posterior distinction in functional insular networks associated with different dimensions of nicotine dependence, with greater dependence linked to weaker connectivity. They may inform therapeutic approaches involving brain stimulation that may elicit differential clinical outcomes depending on the insular subnetwork targeted.
\end{abstract}

Keywords: Nicotine dependence, fMRI, insula, resting state functional connectivity 


\section{Introduction}

The use of combustible tobacco products continues to be a substantial global health problem, causing over 7 million or 1 in 10 deaths world-wide each year ${ }^{1,2}$. Among people who try to quit smoking independently, only 3 to 6 percent successfully stop for 6 to 12 months, with most failing within 8 days ${ }^{3}$. Behavioral and pharmacological treatments for Tobacco Use Disorder (TUD) also have limited success ${ }^{4-7}$, and the need for investigation of novel therapeutic strategies persists. Elucidating the neural mechanisms of nicotine dependence has the potential to advance the treatment of TUD ${ }^{8,9}$, particularly with brain-based approaches, such as targeted brain stimulation ${ }^{10}$. Knowledge of the neural systems underlying nicotine dependence is critical for guiding these treatments.

The insula has been identified as a promising target for brain-based treatments for TUD 11,12, partly due to clinical evidence for its role in smoking behavior. Patients with insula lesions resulting from strokes have exhibited marked reduction in smoking ${ }^{13-16}$. In stroke survivors, damage to the right insula resulted in smoking cessation when assessed one year after discharge from the hospital ${ }^{16}$, and predicted even greater reduction in dependence when combined with damage to the basal ganglia ${ }^{14}$.

Neuroimaging studies have also indicated the importance of the insula in maintenance of cigarette smoking. Cortical thickness of insular sub-regions is negatively related to nicotine dependence ${ }^{17-19}$ and cigarette craving ${ }^{20}$. Resting-state functional connectivity (RSFC) has been used to assess neural systems involved in nicotine dependence ${ }^{21,22}$. Studies that focused on the anterior cingulate cortex (ACC) found a negative relationship between nicotine dependence and connectivity with the striatum ${ }^{23-26}$, and a rodent study showed that insula-frontal connectivity moderates this negative association ${ }^{27}$. Other RSFC studies showed an inverse relationship 
between nicotine dependence and insula-ACC connectivity in humans ${ }^{24,28,29}$.

The insula has been subdivided into three sub-regions that serve distinct functions: dorsal anterior, ventral anterior, and posterior ${ }^{30-32}$. While the posterior portion of the insula connects with sensorimotor integration areas (e.g., pre-motor, supplementary motor cortex), the anterior portion is functionally linked with limbic regions and is a key component of the "salience network", which includes the ACC ${ }^{33,34}$. The anterior portion has generally been shown to serve cognitive and affective functions ${ }^{35,36}$. Further functional distinctions have been made between dorsal and ventral anterior insula connectivity along cognitive and affective domains, respectively $35,37,38$. In addition, dorsal/ventral distinctions have been conceptualized as components of externally- and internally-oriented networks - specifically, the frontoparietal attention network and the default mode network, respectively ${ }^{39,40}$.

Prior studies of RSFC have attempted to distinguish between connectivity of insular subregions and smoking-related behavioral variables ${ }^{24,40-43}$, but only two of them examined RSFC of insula subregions with respect to nicotine dependence ${ }^{24,28}$. In one of these studies, analysis was restricted to the dorsal ACC after demonstration of a difference in anterior insula-ACC connectivity between people who smoked and those who did not; a negative correlation between dependence and anterior insula connectivity with the dorsal ACC was found ${ }^{28}$. In the other study, individuals who smoked and had schizophrenia were compared with individuals who smoked but had no other psychiatric diagnosis ${ }^{24}$. Both groups showed an inverse relationship between nicotine dependence and posterior insula-dorsal ACC connectivity. Despite this initial evidence for distinctions between circuits of insula subregions with respect to dependence, a comprehensive analysis of the contributions of the three major subregions is lacking, leaving open the possibility that RSFC of different insular subregions would be differentially related to 
nicotine dependence. We therefore undertook a comprehensive analysis of correlations with connectivity patterns of these finer-grained subregions in a whole-brain analysis.

The Fagerström Test for Nicotine Dependence (FTND) ${ }^{44}$ is a widely used, validated assessment that is related to biological indices of smoking ${ }^{45,46}$. Neuroimaging studies that evaluate nicotine dependence with the FTND have used a unidimensional scoring system; however, psychometric studies have established that a two-dimensional (two factor) structure is more appropriate ${ }^{47-51}$, with the first factor interpreted as "the degree of urgency to restore nicotine levels to a given threshold after nighttime abstinence" ("morning smoking") and a second factor interpreted as "the persistence with which nicotine levels are maintained at a given threshold during waking hours" (“daytime smoking”) ${ }^{51}$. To date, no neuroimaging studies have examined the neural circuitry underlying this bivariate structure of the FTND, which may provide a more nuanced understanding of nicotine dependence and the neural circuits that support it.

In a group of 47 participants who smoked cigarettes daily, we examined the relationship between nicotine dependence and RSFC of the three major insular sub-regions (ventral anterior, dorsal anterior, and posterior) after overnight abstinence. Considering the literature, we hypothesized that dependence would be negatively correlated both with connectivity between anterior insula subregions and ACC, and with connectivity between the posterior insula and sensory-motor integration regions (e.g., supplementary motor area) (i.e., participants with greater connectivity would show less dependence). To determine the extent to which the two-factor structure reveals greater specificity with respect to the relationship between dependence and insula connectivity, we examined insula RSFC in relation to both the unidimensional scoring (FTND total score) and the two-factors. 


\section{Materials and Methods}

\section{Overview of Experimental Design}

Functional magnetic resonance imaging (fMRI) data were collected during the resting state from adults who smoked cigarettes daily and maintained overnight abstinence before testing. The study was part of a larger investigation on the brain correlates of smoking behavior and took place between September, 2017 and February, 2020. Another report from that study is in press ${ }^{52}$. The study was conducted at the Semel Institute for Neuroscience and Human Behavior at the University of California, Los Angeles (UCLA). All study procedures were approved by the UCLA Institutional Review Board.

\section{Participants}

One-hundred-seventy-nine participants were screened via online and print advertisements. They attended an intake session where they received a detailed explanation of the study procedures, provided written informed consent, and were screened for eligibility. Fiftyone met all study criteria and completed all procedures. Inclusion criteria were as follows: age of 18-45 years, generally good health, self-report of smoking at least 4 cigarettes per day for at least 1 year, and urinary cotinine $\geq 100 \mathrm{ng} / \mathrm{mL}$. Recent smoking history was verified during the intake session using a urine cotinine test (ACCUTEST Urine Cotinine Test, Jant Pharmacal Corp., Encino, CA, score $\geq 3$, cotinine $\geq 100 \mathrm{ng} / \mathrm{mL}$ ). Exclusion criteria were positive urine tests for drugs of abuse other than nicotine or tetrahydrocannabinol, consuming $\geq 10$ alcoholic drinks per week, any current psychiatric disorder other than Tobacco Use Disorder as assessed via the Mini International Neuropsychiatric Interview (MINI) for DSM-5 ${ }^{53}, 54$, history of neurological injury, and using electronic cigarettes, cigars, snuff, or chewing tobacco >3 times a month. 


\section{Verification of Drug and Alcohol Abstinence}

On the testing day, overnight ( 12 hours) abstinence from smoking was verified by a CO level of $<10$ ppm measured with the Micro+ Smokerlyzer® breath CO monitor (Bedford Scientific Ltd., Maidstone, Kent, UK). Abstinence from cocaine, opiates, benzodiazepines, and amphetamines was also verified with a five-panel urine drug test (Drugs of Abuse Test Instaview®, Alfa Scientific Designs Inc., Poway, CA). Alcohol abstinence was verified using a breathalyzer (Alco-Sensor FST®, Intoximeters, Inc., St. Louis, MO). Recent abstinence from cannabis use was verified with the Dräger DrugTest@ 5000 saliva test (Dräger, Inc., Houston, TX).

\section{Self-report of Nicotine Dependence and Analysis Procedures}

Nicotine dependence was measured during the intake session using FTND ${ }^{44}$. The standard total score was computed in addition to the two-factor solution. These factors have been established in the literature using exploratory factor analyses ${ }^{47-50}$ and confirmatory factor analysis ${ }^{51}$, indicating that the six items of the FTND comprise two factors with one item (time to first cigarette of the day) loading on both factors. The first factor included items 1,3 and 5 of the FTND (FTND135) and is interpreted as encompassing "morning smoking", and the second factor is composed of items 1, 2, 4 and 6 (FTND1246), and is interpreted as "daytime smoking" (see Supplementary Table 13 for FTND items). For each participant, we computed a weighted sum of the response to the items in each factor, with weightings based on the mean of the weights reported across previous studies ${ }^{47-50}$. See supplementary materials for analysis details.

We verified that FTND data from our 47 participants matched the two-factor structure determined in the literature on much larger samples. Rather than conducting a confirmatory 
factorial analysis that requires a large number of observations, we performed the same factorial analysis method conducted in the study by Haddock et al. ${ }^{48}$ on 4,042 smokers which used principal component analysis followed by a Varimax rotation. Results of this decomposition (Supplementary Table 13) showed that our data exhibited the same two-factor organization. Namely, FTND135 and FTND1246 explained 54\% of the variance in our study and 51\% in the study of Haddock et al. The average difference in item loading for FTND135 and FTND1246 between both studies was 0.14 and 0.06 , respectively.

\section{Scanning Protocol}

\section{MRI data acquisition:}

All images were acquired on a 3-Tesla PRISMA (Siemens) MRI scanner using a 32channel head coil receiver. The resting state imaging protocol consisted of the continuous acquisition of 738 Echo-planar Image (EPI) volumes over a period of 9 minutes and 50 seconds. A multi-band accelerated EPI pulse sequence (factor 8) was used, allowing us to acquire 72 axial slices during a repetition time (TR) of $800 \mathrm{~ms}$ with a 104x104 matrix. Resolution was 2x2x2 $\mathrm{mm}^{3}$, echo time (TE) was $37 \mathrm{~ms}$, and the flip angle was 52 degrees. Participants were asked to keep their eyes open and to look at a black screen during the resting state scan. The structural T1-weighted images were obtained using a Magnetization Prepared Rapid Gradient Echo (MPRAGE) sequence with the following parameters: isovoxel $0.8 \mathrm{~mm}^{3}, \mathrm{FOV}=240 \times 256 \mathrm{~mm}^{2}$, $\mathrm{TE}=2.24 \mathrm{~ms}, \mathrm{TR}=2400 \mathrm{~ms} ;$ flip angle $=8^{\circ} ; 208$ sagittal slices .

\section{MRI data pre-processing:}

Image preprocessing was mostly conducted with FSL (5.0.9). The initial stages included rigid body realignment to correct for head movements within each scanning run, skull removal, and non-linear registration to the Montreal Neurological Institute (MNI) template. A first motion 
cleaning and noise reduction were performed using a 24-parameter linear regression model that included six motion parameters (3 translational dimensions along $\mathrm{X}, \mathrm{Y}$ and $\mathrm{Z}$ axes and 3 rotational dimensions: "pitch", "roll" and "yaw"), the temporal derivatives of these parameters and the quadratic of all parameters ${ }^{55}$. Mean frame displacement (FD) and the variance of signal change from the average signal (DVARS) of the raw images were estimated. A null sampling distribution of DVARS was used to identify frames with excessive variance at $\mathrm{p}<0.05^{56}$; frames with FD exceeding $0.45 \mathrm{~mm}$ were also flagged. These frames as well as the one located in time just prior ( $\mathrm{t}-1)$ and two just after $(\mathrm{t}+1$ and $\mathrm{t}+2)$ were included in a censoring temporal mask for data interpolation: a least-squares spectral decomposition of the uncensored data was performed to reconstitute data of the censored timepoints see methods in ${ }^{57}$. The uncensored data defined the frequency characteristics of signals that then replaced the censored data. This step aimed at minimizing the contamination of the signal from the censored frames during frequency filtering. The interpolated signal was then demeaned, detrended and filtered using an ideal bandpass filter $(0.009-0.08 \mathrm{~Hz})$. Following band-pass filtering, the interpolated timepoints were finally censored. Participants with more than $50 \%$ frames censored (i.e., those with less than 5 minutes of remaining resting state data) were excluded from the analysis. To reduce the contribution from non-neuronal noise in the data, the minimal number of principle components that explained at least $50 \%$ of the variance of mean signal extracted from white matter and cerebrospinal fluid were evaluated and regressed out from the signal aCompCor $50,{ }^{58}$. Volumes were then spatially smoothed with a Gaussian filter using a 5-mm FWHM kernel. Each voxel was normalized to a mean value of $100(\mathrm{SD}=1)$ to transform the data to Pearson's correlation coefficients (r). All analyses were performed on Linux (CentOS release 6.10) using FSL 5.0.9, MATLAB 8.6, R (version 3.6.0). 


\section{Resting-state fMRI seed-based analysis:}

To minimize bias, we used a statistically conservative voxel-wise whole-brain analytic approach rather than restricting to a priori-selected target regions or networks. On each of the two hemispheres of the brain three insula seeds, which encompassed ventral-anterior, dorsalanterior, and posterior aspects of the insula, were defined for RSFC analyses (Figure 1). To define the anterior insula, we compared anatomical landmarks from a probabilistic atlas ${ }^{59}$ to functional connectivity-based parcellations of the insula ${ }^{30,32}$. From these studies, we defined the ventral anterior insula parcel as the anterior inferior insular cortex (which includes the apex, the limen, and the transverse gyrus). The dorsal anterior insula was defined as the anterior and middle short gyri. The precentral sulcus was used to segment the anterior from the posterior insula. Using these landmarks, we manually determined the anterior insular subdivisions (dorsal and ventral, left and right) from the MNI152 template.

To evaluate the functional connectivity between the insula seeds and other brain regions during the resting state, the time series from each seed was extracted, and its first normalized eigen vector (mean=100, $\mathrm{SD}=1$; to facilitate computation of Pearson's $\mathrm{r}$ ) was used as a regressor in an ordinary least squares linear regression analysis on every voxel (as implemented in film_gls in FEAT). The parameter estimates of the model, corresponding to the Pearson's correlation coefficient (since data were previously normalized), were z-transformed to improve data normality.

The resulting z-transformed images were used in multi-level mixed effects models for group analyses (FLAME1, FEAT) testing for the effect of nicotine dependence on functional connectivity for each seed. Specifically, two separate models were tested. The first model included the total score of the FTND as the independent variable of interest. The second model 
examined separate effects of the two factors of the FTND: FTND135 ("morning smoking") and FTND1246 (“daytime smoking”).

To account for differences in motion during scanning between participants, the mean frame displacement value was included as a covariate in all models in addition to age. Results were cluster-corrected for multiple comparisons using a voxel-height threshold of $\mathrm{p}<0.001$ (Z>3.1) and cluster threshold of $\mathrm{p}<0.05$ as recommended per Eklund et al ${ }^{60}$. The coordinates reported here correspond to the peak voxel within a given cluster in MNI coordinate space. All post-hoc tests on data extracted from statistical maps were conducted using $\mathrm{R}^{61}$. For each participant, data were extracted and averaged from voxels within clusters that survived the cluster-correction threshold (voxel height $=\mathrm{Z}>3.1$, cluster extent $=\mathrm{P}<0.05)$.

\section{Results}

\section{Participant Characteristics}

Fifty-one adults who endorsed daily cigarette smoking completed overnight ( 12 hours) abstinence from smoking. Of the 51 participants tested, four were excluded for excessive motion during fMRI, as revealed by the number of flagged volumes exceeding DVARS and/or FD thresholds after data preprocessing ( $>50 \%)$. The final sample included 47 individuals ( 24 female) with a mean age of $33.3(\mathrm{SD}=7.2)$ years. On average, they smoked $11.4(\mathrm{SD}=4.5)$ cigarettes per day with a smoking history of $8.1(\mathrm{SD}=6.0)$ pack years. Nicotine dependence varied from low to high levels (mean FTND total scores of 4.0, $\mathrm{SD}=2.0$ ). Other participant characteristics, including ethnicity, race, alcohol, and cannabis consumption, are shown in Table 1.

\section{Nicotine dependence}

Testing the association of the FTND total score with functional connectivity between the insula seeds and the rest of the brain revealed negative relationships in all cases. Specifically, 
FTND was negatively correlated with: (1) connectivity of the left ventral anterior insula (lvaInsula) and the precuneus (X=-12, Y=-70, $\mathrm{Z}=48$; Figure 2A, B), (2) connectivity of the right dorsal anterior insula (rdaInsula) and a precuneus target with very similar coordinates $(\mathrm{X}=-10$, $\mathrm{Y}=-72 ; \mathrm{Z}=50$ ) (Figure 2A, B, C), and (3) connectivity between the left posterior insula (lpInsula) seed and the left posterior aspect of the putamen $(X=-28, Y=-12, Z=2)$ (Figure 3A, C, D).

The second model, which tested for separate effects of the two FTND factors, revealed a negative relationship between "daytime smoking" (FTND1246) and connectivity of the lpInsula and the same left posterior putamen area found in analysis of the FTND total score $(\mathrm{X}=-30, \mathrm{Y}=-$ 14; Z=2; Figure 3B, C, D). No other significant relationships were observed, including any with the "morning smoking" factor (FTND135).

\section{Post hoc analyses of associations with nicotine dependence}

The results presented above were determined in whole-brain voxel-wise analyses that involved a conservative correction for multiple comparisons. We noted the asymmetry of the insular seeds that yielded the patterns observed and aimed to explore the extent of this asymmetry using post-hoc region-of-interest analyses. Accordingly, we conducted region-ofinterest analyses testing for relationships between dependence and connectivity between all four anterior insula seeds (tested separately) and the left precuneus cluster as well as the two posterior insula seeds and the left putamen cluster. These analyses were conducted using the same two linear models - one with FTND total as independent variable and another with FTND135 and FTND1246. The results indicated that functional connectivity of all four anterior insula seeds (not only the rdaInsula and lvaInsula) with the precuneus was negatively correlated with the total FTND score at $p s<0.001$ (Supplementary Tables 1-4) and that both left and right posterior insula 
connectivity with the left posterior putamen had the same modulatory pattern $\left(p s<10^{-5}\right)$ (Supplementary Tables 5 and 6).

The test of the two-factor model indicated that, similar to findings for lpInsula, rpInsula connectivity with the left posterior putamen was also modulated by the "daytime smoking" factor (FTND1246) $\left(p<2 \times 10^{-6}\right)$ (Supplementary Tables 7 and 8). Connectivity of all anterior seeds of the insula with the precuneus showed significant negative relationships with both factors, except for the rvaInsula $(\mathrm{p}=0.08)$ (Supplementary Tables 9-12). Table 2 summarizes these post hoc results.

\section{Discussion}

This study showed that nicotine dependence was negatively correlated with two distinct functional connectivity patterns across different insular subregions. Taken together, the combined results of a priori whole-brain and post-hoc region-of-interest analyses indicated that nicotine dependence is associated with connectivity of the anterior insula and the right precuneus and with connectivity of the posterior insula and the left posterior putamen. Moreover, when examining two separate aspects of dependence (as defined by the two-factor model), anterior insula-right precuneus connectivity was related to both "morning smoking" and "daytime smoking" whereas posterior insula-left putamen connectivity was only related to "daytime smoking." Thus, different dimensions of dependence apparently are related to connectivity of separate insular subregions.

This separation of anterior vs. posterior insula functional connectivity is somewhat consistent with functional distinctions observed along the anterior/posterior insula axis (affective-cognitive/sensorimotor, respectively) ${ }^{35}$. Although we hypothesized a relationship between dependence and connectivity of anterior insula subregions with limbic regions and 
components of the salience network (i.e. ACC), we found a relationship with connectivity to the precuneus, a region that is not included among limbic or salience network structures but is involved in cognitive and affective function. The precuneus has been associated with selfreferential processing, empathy, and episodic memory retrieval ${ }^{62}$, and has a central role in the default mode network ${ }^{63-65}$. In studies of substance use, the precuneus is among the primary brain regions activated during smoking cue-induced neural reactivity, as indicated by meta-analyses ${ }^{66}$. Precuneus activation during presentation of smoking- and alcohol-related cues is positively associated with nicotine and alcohol dependence, respectively ${ }^{67}$. Moreover, in individuals who smoke cigarettes, anterior insula-precuneus connectivity, a similar connectivity pattern to that observed in the current study, was correlated with cue-induced craving ${ }^{68}$. Combined with these findings from prior studies, those presented here suggest a role for the interaction of the precuneus with the anterior insula in maintenance of nicotine dependence, especially aspects that involve self-referential processes.

The relationship between nicotine dependence and posterior insula-left posterior putamen connectivity is consistent with the view that the insula supports an embodied experience of addiction via integration of interoceptive signals ${ }^{69}$, in light of the role of the posterior insula in interoception ${ }^{70-72}$ and evidence for a role of the putamen in habitual stimulus-response associations ${ }^{73}$. A study of stroke patients comparing those with basal ganglia lesions (including the putamen), and those with both insula and basal ganglia lesions, showed that patients with both lesions had a greater disruption of smoking after their stroke than patients with basal ganglia lesions alone ${ }^{14}$, providing evidence for the relevance of insula-basal ganglia interactions in maintaining dependence. Our functional connectivity finding is also supported by a structural connectivity study that mapped insular subregions to subcortical regions, indicating that of the 
subcortical regions tested (thalamus, amygdala, hippocampus, putamen, globus pallidus, caudate nucleus, and nucleus accumbens), the putamen had the most connections with the posterior insula ${ }^{74}$. Association of weaker posterior insula-left putamen functional connectivity with greater nicotine dependence is in line with meta-analytic findings of less engagement of striatal regions in response to smoking-related and non-smoking reward-related cues with greater nicotine dependence ${ }^{75}$. The latter findings suggest that weak posterior insula-putamen connectivity may also be related to disrupted reward-related responses in the striatum.

This study is the first to examine neuroimaging markers of nicotine dependence with respect to the widely established two-factor characterization of dependence measured using the FTND. That posterior insula-left putamen connectivity uniquely correlated with the "daytime smoking" factor, and not "morning smoking", suggests that this functional circuit especially serves persistence in maintaining nicotine levels throughout the day. Given that some of the items included in this factor may be considered as assessments of self-control (e.g., "Do you find it difficult to keep from smoking in places where it is not allowed?"), weakened connectivity of this circuit may lead to disruption of interoceptive signals that support self-control behavior. The relationship between anterior insula-precuneus connectivity and both factors suggests that this functional circuit is important for multiple dimensions of dependence.

Our results are only partially consistent with previous insula RSFC studies of nicotine dependence, which focused on the ACC and found significant associations involving the ACC. A previous study of non-deprived young participants (15-24 years of age) found a negative relationship between anterior insula-ACC connectivity and nicotine dependence ${ }^{28}$; but ACC was selected as the connectivity target post-hoc, based on an analysis that compared anterior insula connectivity of individuals who did or did not smoke and found greater connectivity with the 
ACC as a primary group difference. Another study similarly found a negative association between dependence and insula-ACC connectivity ${ }^{29}$, but it used the whole, bilateral insula as a seed, eliminating the possibility of differentiating effects of insular subregions. Lastly, a third study found a negative relationship between nicotine dependence and connectivity between the posterior insula and ACC in individuals with schizophrenia and also those without psychiatric diagnoses ${ }^{24}$. It is possible that the current study did not find significant results involving the ACC because we used a whole-brain voxel-wise approach, with strict multiple comparison correction, to determine which connectivity targets across the whole brain were associated with dependence. Another reason for the absence of a positive finding regarding the ACC may be our use of rigorous motion-cleaning approaches $57,76,77$, which are considered essential for removing artifacts in the data that may lead to spurious correlations ${ }^{78}$.

Brain stimulation has been considered as a promising therapy for smoking cessation ${ }^{79,80}$, and studies targeting the insula have demonstrated mixed success in affecting smoking-related variables, such as craving and withdrawal ${ }^{10-12}$. By highlighting the relevance of two insular functional connectivity patterns, which are weaker in strength in those who have greater nicotine dependence, we provide additional relevant targets for future stimulation studies. For example, stimulation studies may not only choose to target anterior and posterior insula, but also the right precuneus and left posterior putamen, with the aim of increasing the strength of the relevant functional connectivity patterns identified in the current study.

The current study is limited in that it cannot determine whether weak insular connectivity is a cause or consequence of nicotine dependence although pre-clinical studies have suggested a potential causal role of insular connectivity on dependence. In a data-driven study of rats, Hsu et al. ${ }^{81}$ showed that an insular-frontal network module was predictive of later nicotine dependence. 
However, it remains to be determined if a similar finding will emerge from longitudinal human studies, such as the Adolescent Brain Development Study (ABCD) study ${ }^{82}$.

Overall, this study provides evidence for the association of nicotine dependence with weak functional connectivity of distinct insula subregions, namely, anterior insula-right precuneus and posterior insula-left putamen. This regional dissociation within the insula highlights the heterogeneity of the insula with respect to neural processes involved in maintenance of smoking and suggests multiple potential targets for brain-based therapies that address nicotine addiction. 


\section{Funding and Disclosure:}

This research was supported, in part, by a grant from the National Institute on Drug Abuse (NIDA) (R37 DA044467, EDL) and endowments from the Thomas P. and Katherine K. Pike Chair in Addiction Studies and the Marjorie M. Greene Trust (EDL). Dr. Perez Diaz is supported by a Ruth L. Kirschstein Postdoctoral Individual National Research Award from NIDA (F32 DA049500-01A1). We acknowledge the Canada Research Chairs program (Dr. Tyndale, the Canada Research Chair in Pharmacogenomics). Dr. Tyndale has consulted for Quinn Emanuel and Ethismos Research Inc. All other authors declare no conflicts of interest.

\section{Acknowledgements:}

The authors thank Ms. Andrea Donis, Ms. Diana Paez, Ms. Citlaly Cahuantzi, Ms. Tinisha Sakhrani, and Mr. Hector Diaz, whose contribution to data collection helped make this work possible.

\section{Author Contributions:}

All authors were involved in designing the study and contributed to writing the manuscript. Drs. Ghahremani and Perez Diaz acquired the data. Drs. Pochon, Ghahremani, Perez Diaz and Dean were responsible for data analysis. Dr. Tyndale contributed to decisions regarding participant characterization. Drs. Ghahremani, Pochon, and London drafted the manuscript. As principal investigator of the study, Dr. London is accountable for all aspects of the work, including its accuracy and integrity. All authors have reviewed and approve the final version of this manuscript. 
Data Availability:

All self-report, toxicology, and summary fMRI data discussed in this manuscript, as well as the code used for statistical analyses, are publicly available from the Open Science Framework web site under project title, "Nicotine dependence and functional connectivity of insular cortex subregions" (https://osf.io/24tkf/). 


\section{References}

1. Reitsma MB, Fullman N, Ng M, et al. Smoking prevalence and attributable disease burden in 195 countries and territories, 1990-2015: a systematic analysis from the Global Burden of Disease Study 2015. The Lancet. 2017;389(10082):1885-1906.

2. World Health Organization. WHO report on the global tobacco epidemic, 2017: monitoring tobacco use and prevention policies. https://www.who.int/tobacco/global report/2017/en/

3. Hughes JR, Keely J, Naud S. Shape of the relapse curve and long-term abstinence among untreated smokers. Addiction. Jan 2004;99(1):29-38. doi:10.1111/j.1360-0443.2004.00540.x 4. Martín Cantera C, Puigdomènech E, Ballvé JL, et al. Effectiveness of multicomponent interventions in primary healthcare settings to promote continuous smoking cessation in adults: a systematic review. BMJ Open. 2015;5(10):e008807. doi:10.1136/bmjopen-2015-008807

5. Babb S, Malarcher A, Schauer G, Asman K, Jamal A. Quitting smoking among adultsUnited States, 2000-2015. Morbidity and Mortality Weekly Report. 2017;65(52):1457-1464.

6. Rosen LJ, Galili T, Kott J, Goodman M, Freedman LS. Diminishing benefit of smoking cessation medications during the first year: a meta-analysis of randomized controlled trials. Addiction. May 2018;113(5):805-816. doi:10.1111/add.14134

7. Gómez-Coronado N, Walker AJ, Berk M, Dodd S. Current and Emerging Pharmacotherapies for Cessation of Tobacco Smoking. Pharmacotherapy: The Journal of Human Pharmacology and Drug Therapy. 2018;38(2):235-258.

doi:https://doi.org/10.1002/phar.2073

8. D'Souza MS, Markou A. Neuronal mechanisms underlying development of nicotine dependence: implications for novel smoking-cessation treatments. Addiction science \& clinical practice. Jul 2011;6(1):4-16.

9. United States Public Health Service Office of the Surgeon General; National Center for Chronic Disease Prevention and Health Promotion (US) Office on Smoking and Health. Chapter 3. New Biological Insights into Smoking Cessation. Smoking Cessation: A Report of the Surgeon General [Internet]. US Department of Health and Human Services; 2020.

10. Antonelli M, Fattore L, Sestito L, Di Giuda D, Diana M, Addolorato G. Transcranial Magnetic Stimulation: A review about its efficacy in the treatment of alcohol, tobacco and cocaine addiction. Addictive behaviors. Mar 2021;114:106760.

doi:10.1016/j.addbeh.2020.106760

11. Ibrahim C, Rubin-Kahana DS, Pushparaj A, et al. The insula: a brain stimulation target for the treatment of addiction. Frontiers in pharmacology. 2019;10:720.

12. Regner MF, Tregellas J, Kluger B, Wylie K, Gowin JL, Tanabe J. The insula in nicotine use disorder: Functional neuroimaging and implications for neuromodulation. Neuroscience \& Biobehavioral Reviews. 2019/08/01/ 2019;103:414-424.

doi:https://doi.org/10.1016/j.neubiorev.2019.06.002

13. Abdolahi A, Williams GC, Benesch CG, et al. Damage to the insula leads to decreased nicotine withdrawal during abstinence. Addiction. 2015;110(12):1994-2003.

14. Gaznick N, Tranel D, McNutt A, Bechara A. Basal ganglia plus insula damage yields stronger disruption of smoking addiction than basal ganglia damage alone. nicotine \& tobacco research. 2014;16(4):445-453.

15. Naqvi NH, Rudrauf D, Damasio H, Bechara A. Damage to the insula disrupts addiction to cigarette smoking. Science. 2007;315(5811):531-534. 
16. Suñer-Soler R, Grau A, Gras ME, et al. Smoking cessation 1 year poststroke and damage to the insular cortex. Stroke. 2012;43(1):131-136.

17. Lin F, Wu G, Zhu L, Lei H. Region-Specific Changes of Insular Cortical Thickness in Heavy Smokers. Frontiers in human neuroscience. 2019;13:265. doi:10.3389/fnhum.2019.00265 18. Wang C, Huang P, Shen Z, et al. Gray matter volumes of insular subregions are not correlated with smoking cessation outcomes but negatively correlated with nicotine dependence severity in chronic smokers. Neuroscience Letters. 2019/03/23/ 2019;696:7-12. doi:https://doi.org/10.1016/j.neulet.2018.12.013

19. Morales AM, Ghahremani D, Kohno M, Hellemann GS, London ED. Cigarette exposure, dependence, and craving are related to insula thickness in young adult smokers.

Neuropsychopharmacology. Jul 2014;39(8):1816-22. doi:10.1038/npp.2014.48

20. Perez Diaz M, Pochon J-B, Ghahremani DG, et al. Sex Differences in the Association of Cigarette Craving With Insula Structure. International Journal of Neuropsychopharmacology. 2021;

21. Fedota JR, Stein EA. Resting-state functional connectivity and nicotine addiction: prospects for biomarker development. Annals of the New York Academy of Sciences. 2015;1349(1):64.

22. Sutherland MT, McHugh MJ, Pariyadath V, Stein EA. Resting state functional connectivity in addiction: Lessons learned and a road ahead. Neuroimage. 2012;62(4):22812295.

23. Hong LE, Gu H, Yang Y, et al. Association of nicotine addiction and nicotine's actions with separate cingulate cortex functional circuits. Archives of general psychiatry. Apr 2009;66(4):431-41. doi:10.1001/archgenpsychiatry.2009.2

24. Moran LV, Sampath H, Stein EA, Hong LE. Insular and anterior cingulate circuits in smokers with schizophrenia. Schizophrenia Research. 2012/12/01/ 2012;142(1):223-229. doi:https://doi.org/10.1016/j.schres.2012.08.033

25. Li S, Yang Y, Hoffmann E, Tyndale RF, Stein EA. CYP2A6 Genetic Variation Alters Striatal-Cingulate Circuits, Network Hubs, and Executive Processing in Smokers. Biological Psychiatry. 2017/04/01/ 2017;81(7):554-563. doi:https://doi.org/10.1016/j.biopsych.2016.09.013 26. Hong LE, Hodgkinson CA, Yang Y, et al. A genetically modulated, intrinsic cingulate circuit supports human nicotine addiction. Proceedings of the National Academy of Sciences of the United States of America. Jul 27 2010;107(30):13509-14. doi:10.1073/pnas.1004745107 27. Keeley RJ, Hsu LM, Brynildsen JK, Lu H, Yang Y, Stein EA. Intrinsic differences in insular circuits moderate the negative association between nicotine dependence and cingulatestriatal connectivity strength. Neuropsychopharmacology. May 2020;45(6):1042-1049. doi:10.1038/s41386-020-0635-X

28. Bi Y, Yuan K, Guan Y, et al. Altered resting state functional connectivity of anterior insula in young smokers. Brain imaging and behavior. Feb 2017;11(1):155-165. doi:10.1007/s11682-016-9511-z

29. Zhou S, Xiao D, Peng P, et al. Effect of smoking on resting-state functional connectivity in smokers: An fMRI study. Respirology (Carlton, Vic). Aug 2017;22(6):1118-1124. doi:10.1111/resp.13048

30. Chang LJ, Yarkoni T, Khaw MW, Sanfey AG. Decoding the role of the insula in human cognition: functional parcellation and large-scale reverse inference. Cerebral cortex. 2013;23(3):739-749. 
31. Cauda F, D'Agata F, Sacco K, Duca S, Geminiani G, Vercelli A. Functional connectivity of the insula in the resting brain. Neuroimage. Mar $12011 ; 55(1): 8-23$.

doi:10.1016/j.neuroimage.2010.11.049

32. Deen B, Pitskel NB, Pelphrey KA. Three systems of insular functional connectivity identified with cluster analysis. Cerebral cortex. 2011;21(7):1498-1506.

33. Seeley WW, Menon V, Schatzberg AF, et al. Dissociable intrinsic connectivity networks for salience processing and executive control. Journal of Neuroscience. 2007;27(9):2349-2356.

34. Uddin LQ. Salience network of the human brain. Academic press; 2016.

35. Kurth F, Zilles K, Fox PT, Laird AR, Eickhoff SB. A link between the systems: functional differentiation and integration within the human insula revealed by meta-analysis. Brain structure \& function. Jun 2010;214(5-6):519-34. doi:10.1007/s00429-010-0255-z

36. Gu X, Liu X, Van Dam NT, Hof PR, Fan J. Cognition-emotion integration in the anterior insular cortex. Cerebral cortex (New York, NY : 1991). Jan 2013;23(1):20-7.

doi:10.1093/cercor/bhr367

37. Touroutoglou A, Hollenbeck M, Dickerson BC, Feldman Barrett L. Dissociable largescale networks anchored in the right anterior insula subserve affective experience and attention. NeuroImage. 2012/05/01/ 2012;60(4):1947-1958. doi:https://doi.org/10.1016/j.neuroimage.2012.02.012

38. Wager TD, Barrett LF. From affect to control: Functional specialization of the insula in motivation and regulation. BioRxiv. 2017:102368.

39. Wang Y, Zhu L, Zou Q, et al. Frequency dependent hub role of the dorsal and ventral right anterior insula. Neuroimage. Jan 15 2018;165:112-117.

doi:10.1016/j.neuroimage.2017.10.004

40. Janes AC, Krantz NL, Nickerson LD, Frederick BB, Lukas SE. Craving and Cue Reactivity in Nicotine-Dependent Tobacco Smokers Is Associated With Different Insula Networks. Biological psychiatry Cognitive neuroscience and neuroimaging. Jan 2020;5(1):7683. doi:10.1016/j.bpsc.2019.09.005

41. Addicott MA, Sweitzer MM, Froeliger B, Rose JE, McClernon FJ. Increased Functional Connectivity in an Insula-Based Network is Associated with Improved Smoking Cessation Outcomes. Neuropsychopharmacology. 2015/10/01 2015;40(11):2648-2656.

doi:10.1038/npp.2015.114

42. Ghahremani DG, Pochon J-B, Perez Diaz M, Tyndale RF, Dean AC, London ED. Functional connectivity of the anterior insula during withdrawal from cigarette smoking. Neuropsychopharmacology. in press;

43. Fedota JR, Ding X, Matous AL, et al. Nicotine Abstinence Influences the Calculation of Salience in Discrete Insular Circuits. Biological Psychiatry: Cognitive Neuroscience and Neuroimaging. 2018/02/01/ 2018;3(2):150-159. doi:https://doi.org/10.1016/j.bpsc.2017.09.010 44. Fagerström K. Determinants of tobacco use and renaming the FTND to the Fagerström Test for Cigarette Dependence. Nicotine \& tobacco research. 2011;14(1):75-78.

45. Weinberger AH, Reutenauer EL, Allen TM, et al. Reliability of the Fagerström Test for Nicotine Dependence, Minnesota Nicotine Withdrawal Scale, and Tiffany Questionnaire for Smoking Urges in Smokers with and without Schizophrenia. Drug and Alcohol Dependence. 2007/01/12/ 2007;86(2):278-282. doi:https://doi.org/10.1016/j.drugalcdep.2006.06.005

46. Kozlowski LT, Porter CQ, Orleans CT, Pope MA, Heatherton T. Predicting smoking cessation with self-reported measures of nicotine dependence: FTQ, FTND, and HSI. Drug and 
Alcohol Dependence. 1994/02/01/ 1994;34(3):211-216. doi:https://doi.org/10.1016/03768716(94)90158-9

47. Chabrol H, Niezborala M, Chastan E, Montastruc J-L, Mullet E. A study of the psychometric properties of the Fagestrom Test for Nicotine Dependence. Addictive behaviors. 2003;28(8):1441-1445.

48. Haddock CK, Lando H, Klesges RC, Talcott GW, Renaud EA. A study of the psychometric and predictive properties of the Fagerström Test for Nicotine Dependence in a population of young smokers. Nicotine \& Tobacco Research. 1999;1(1):59-66.

49. Payne TJ, Smith PO, McCracken LM, McSherry WC, Antony MM. Assessing nicotine dependence: A comparison of the Fagerström Tolerance Questionnaire (FTQ) with the Fagerström Test for Nicotine Dependence (FTND) in a clinical sample. Addictive behaviors. 1994;19(3):307-317.

50. Radzius A, Epstein DH, Gorelick DA, et al. A factor analysis of the Fagerström Test for Nicotine Dependence (FTND). Nicotine \& Tobacco Research. 2003;5(2):255-260.

51. Richardson CG, Ratner PA. A confirmatory factor analysis of the Fagerstrom Test for Nicotine Dependence. Addictive behaviors. 2005;30(4):697-709.

52. Perez Diaz M, Pochon J-B, Ghahremani DG, et al. Sex differences in the association of cigarette craving with insula structure. Int J Neuropsychopharmacol accepted 2021;

53. Sheehan DV, Lecrubier Y, Sheehan KH, et al. The Mini-International Neuropsychiatric Interview (MINI): the development and validation of a structured diagnostic psychiatric interview for DSM-IV and ICD-10. The Journal of clinical psychiatry. 1998;

54. Hergueta T, Weiller E. Evaluating depressive symptoms in hypomanic and manic episodes using a structured diagnostic tool: validation of a new Mini International Neuropsychiatric Interview (MINI) module for the DSM-5'With Mixed Features' specifier. International journal of bipolar disorders. 2013;1(1):21.

55. Satterthwaite TD, Elliott MA, Gerraty RT, et al. An improved framework for confound regression and filtering for control of motion artifact in the preprocessing of resting-state functional connectivity data. Neuroimage. 2013;64:240-256.

56. Afyouni S, Nichols TE. Insight and inference for DVARS. NeuroImage. 2018;172:291312.

57. Power JD, Mitra A, Laumann TO, Snyder AZ, Schlaggar BL, Petersen SE. Methods to detect, characterize, and remove motion artifact in resting state fMRI. Neuroimage. 2014;84:320341.

58. Muschelli J, Nebel MB, Caffo BS, Barber AD, Pekar JJ, Mostofsky SH. Reduction of motion-related artifacts in resting state fMRI using aCompCor. Neuroimage. 2014;96:22-35.

59. Faillenot I, Heckemann RA, Frot M, Hammers A. Macroanatomy and 3D probabilistic atlas of the human insula. NeuroImage. 2017;150:88-98.

60. Eklund A, Nichols TE, Knutsson H. Cluster failure: Why fMRI inferences for spatial extent have inflated false-positive rates. Proceedings of the National Academy of Sciences. 2016;113(28):7900-7905. doi:10.1073/pnas.1602413113

61. $R$ : A Language and Environment for Statistical Computing. R Foundation for Statistical Computing; 2019. https://www.R-project.org

62. Cavanna AE, Trimble MR. The precuneus: a review of its functional anatomy and behavioural correlates. Brain : a journal of neurology. Mar 2006;129(Pt 3):564-83. doi:10.1093/brain/aw1004 
63. Utevsky AV, Smith DV, Huettel SA. Precuneus is a functional core of the default-mode network. Journal of Neuroscience. 2014;34(3):932-940.

64. Fransson P, Marrelec G. The precuneus/posterior cingulate cortex plays a pivotal role in the default mode network: Evidence from a partial correlation network analysis. Neuroimage. 2008;42(3):1178-1184.

65. Li R, Utevsky AV, Huettel SA, et al. Developmental maturation of the precuneus as a functional core of the default mode network. Journal of cognitive neuroscience. 2019;31(10):1506-1519.

66. Engelmann JM, Versace F, Robinson JD, et al. Neural substrates of smoking cue reactivity: a meta-analysis of fMRI studies. Neuroimage. Mar 2012;60(1):252-62. doi:10.1016/j.neuroimage.2011.12.024

67. Courtney KE, Ghahremani DG, London ED, Ray LA. The association between cuereactivity in the precuneus and level of dependence on nicotine and alcohol. Drug and alcohol dependence. 2014;141:21-26.

68. Moran-Santa Maria MM, Hartwell KJ, Hanlon CA, et al. Right anterior insula connectivity is important for cue-induced craving in nicotine-dependent smokers. Addiction Biology. 2015;20(2):407-414. doi:https://doi.org/10.1111/adb.12124

69. Paulus MP, Stewart JL. Interoception and drug addiction. Neuropharmacology. Jan 2014;76 Pt B(0 0):342-50. doi:10.1016/j.neuropharm.2013.07.002

70. Craig AD. Interoception: the sense of the physiological condition of the body. Current opinion in neurobiology. Aug 2003;13(4):500-5. doi:10.1016/s0959-4388(03)00090-4

71. Critchley HD, Wiens S, Rotshtein P, Ohman A, Dolan RJ. Neural systems supporting interoceptive awareness. Nature neuroscience. Feb 2004;7(2):189-95. doi:10.1038/nn1176 72. Craig AD. How do you feel? Interoception: the sense of the physiological condition of the body. Nature reviews Neuroscience. Aug 2002;3(8):655-66. doi:10.1038/nrn894

73. Yin $\mathrm{HH}$, Knowlton BJ. The role of the basal ganglia in habit formation. Nature reviews Neuroscience. Jun 2006;7(6):464-76. doi:10.1038/nrn1919

74. Ghaziri J, Tucholka A, Girard G, et al. Subcortical structural connectivity of insular subregions. Scientific Reports. 2018/06/05 2018;8(1):8596. doi:10.1038/s41598-018-26995-0

75. Lin X, Deng J, Shi L, et al. Neural substrates of smoking and reward cue reactivity in smokers: a meta-analysis of fMRI studies. Translational Psychiatry. 2020/03/17 2020;10(1):97. doi:10.1038/s41398-020-0775-0

76. Power JD, Schlaggar BL, Petersen SE. Recent progress and outstanding issues in motion correction in resting state fMRI. Neuroimage. 2015;105:536-551.

77. Siegel JS, Power JD, Dubis JW, et al. Statistical improvements in functional magnetic resonance imaging analyses produced by censoring high-motion data points. Human brain mapping. 2014;35(5):1981-1996.

78. Power JD, Barnes KA, Snyder AZ, Schlaggar BL, Petersen SE. Spurious but systematic correlations in functional connectivity MRI networks arise from subject motion. Neuroimage. 2012;59(3):2142-2154.

79. Grall-Bronnec M, Sauvaget A. The use of repetitive transcranial magnetic stimulation for modulating craving and addictive behaviours: A critical literature review of efficacy, technical and methodological considerations. Neuroscience \& Biobehavioral Reviews. 2014/11/01/ 2014;47:592-613. doi:https://doi.org/10.1016/j.neubiorev.2014.10.013 
80. Wing VC, Barr MS, Wass CE, et al. Brain Stimulation Methods to Treat Tobacco Addiction. Brain Stimulation. 2013/05/01/ 2013;6(3):221-230.

doi:https://doi.org/10.1016/j.brs.2012.06.008

81. Hsu LM, Keeley RJ, Liang X, et al. Intrinsic Insular-Frontal Networks Predict Future Nicotine Dependence Severity. The Journal of neuroscience : the official journal of the Society for Neuroscience. Jun 19 2019;39(25):5028-5037. doi:10.1523/jneurosci.0140-19.2019 82. Bjork JM, Straub LK, Provost RG, Neale MC. The ABCD study of neurodevelopment: Identifying neurocircuit targets for prevention and treatment of adolescent substance abuse. Current treatment options in psychiatry. 2017;4(2):196-209. 
Table 1. Participant Characteristics $(n=47)$

$\operatorname{Sex}(F / M) \quad n=24 / n=23$

Ethnicity

Hispanic/Latino (12.8\%)

Non-Hispanic/Latino (83\%)

Unknown (4.3\%)

Race

African-American (23.4\%)

Asian $(2.1 \%)$

Caucasian $(59.6 \%)$

Mixed $(8.5 \%)$

Pacific Islander (4.3\%)

Unknown $(2.1 \%)$

Age (years)

Mean (SD)

Nicotine Dependence ${ }^{\mathrm{a}}$

$33.3 \quad$ (7.2)

Tobacco / current use, cigarettes/day

$11.4 \quad(4.5)$

Tobacco / lifetime exposure, pack-years

$8.1 \quad(6.0)$

Alcohol consumption (drinks/week)

$\mathrm{n}=24$ (>1 drink / week)

$4.3 \quad$ (1.99)

Cannabis consumption (grams/week),

$$
\mathrm{n}=13 \text { (>1 gram / week) } \quad 3.92 \quad \text { (5.19) }
$$

${ }^{\mathrm{a}}$ Fagerström Test of Nicotine Dependence 
Table 2. Summary of post-hoc region of interest analyses of the right precuneus and left putamen for testing contra-lateral insula seeds. Results of each post-hoc linear model is presented in Supplementary Tables 1-12.

\begin{tabular}{llll} 
& & \multicolumn{2}{c}{ Right precuneus } \\
\hline \multirow{2}{*}{ Insula seed } & FTND & "Morning & "Daytime \\
& Total & Smoking", & Smoking"b \\
\hline left ventral anterior insula & $* * *$ & $*$ & $* *$ \\
left dorsal anterior insula & $* * *$ & $*$ & $*$ \\
right ventral anterior insula & $* * *$ & $*$ & \\
right dorsal anterior insula & $* * *$ & $* *$ & $*$ \\
\hline
\end{tabular}

\begin{tabular}{llll} 
& \multicolumn{3}{c}{ Left putamen } \\
\hline \multirow{2}{*}{ Insula seed } & FTND & "Morning & "Morning \\
& Total & Smoking"a & Smoking", \\
\hline left posterior insula & $* * *$ & $* * *$ \\
right posterior insula & $* * *$ & $* * *$ \\
\hline$*=\mathrm{p}<0.05, * * * \mathrm{P}<0.01, * * *=\mathrm{P}<0.001$ & & $*$
\end{tabular}

$*=\mathrm{p}<0.05, * *=\mathrm{P}<0.01, * * *=\mathrm{P}<0.001$

a "Morning Smoking" was associated with FTND items 1, 3, and 5

b "Daytime Smoking" was associated with FTND items 1, 2, 4, and 6 


\section{Figure legends}

Figure 1. Insula sub-regions used as seeds for resting state functional connectivity analyses. Red - ventral anterior insula, Blue - dorsal anterior insula, Green - posterior Insula.

Figure 2. Relationship between nicotine dependence (FTND total score) with anterior insula-precuneus connectivity. A. Thresholded statistical maps indicating functional connectivity of left ventral anterior (hot colors) and right ventral dorsal (cool colors) with a cluster in the left precuneus correlating with FTND total score (voxel threshold: Z>3.1, cluster threshold: $\mathrm{P}<0.05)$. Brain images are presented in neurological convention (right=right). $\mathbf{B}$. Scatterplots of data extracted from the left precuneus cluster from individual participants are shown along with linear fits to illustrate the negative direction of the relationship between FTND and functional connectivity.

Figure 3. Relationship between nicotine dependence with posterior insula-left putamen connectivity. Thresholded statistical maps indicating functional connectivity of left posterior insula with a cluster in the left posterior putamen correlating with (A) FTND total score and (B) the "daytime smoking" factor (FTND1246) from the two-factor model of FTND (voxel threshold: $Z>3.1$, cluster threshold: $P<0.05)$. C. Scatterplots of data extracted from the left putamen clusters from individual participants are shown along with linear fits to illustrate the negative direction of the relationship between the FTND measures and functional connectivity. 
bioRxiv preprint doi: https://doi.org/10.1101/2021.07.07.451360; this version posted July 8, 2021. The copyright holder for this preprint (which was not certified by peer review) is the author/funder. All rights reserved. No reuse allowed without permission.

\section{Figures}

\section{Figure 1}

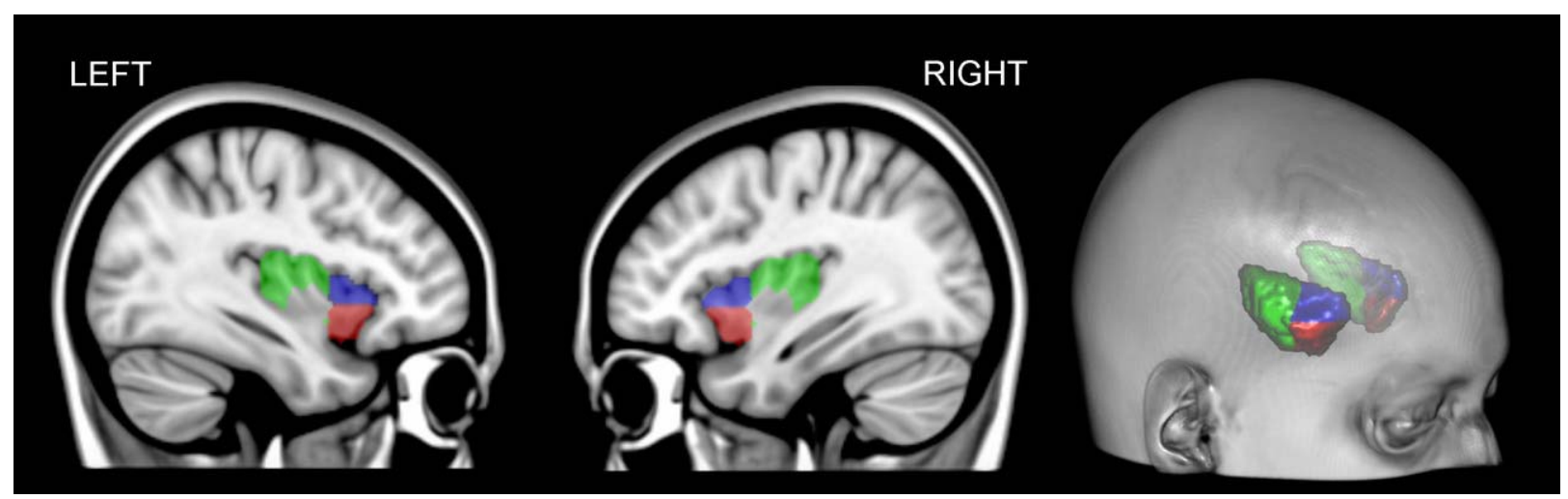


Figure 2

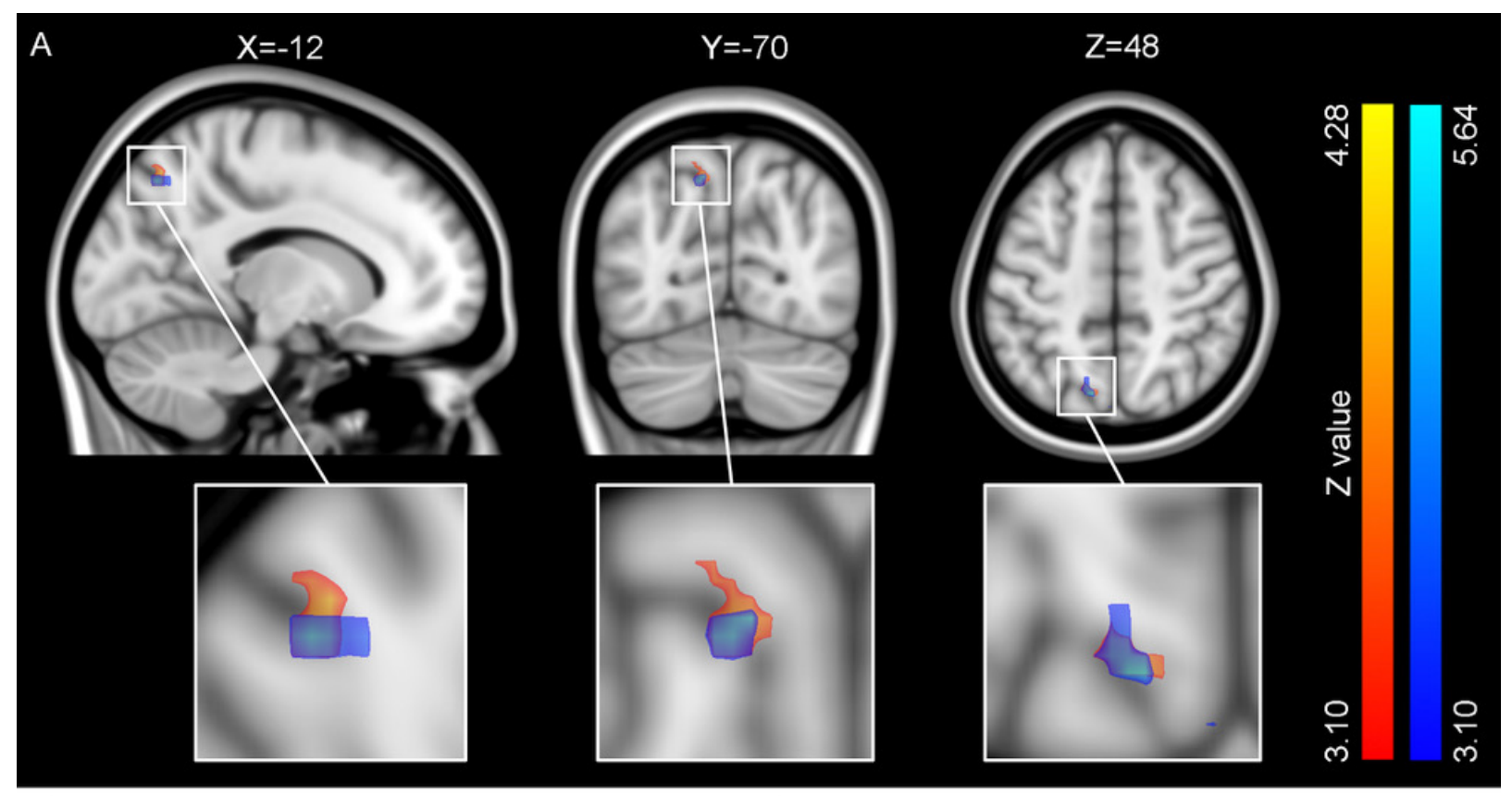

B

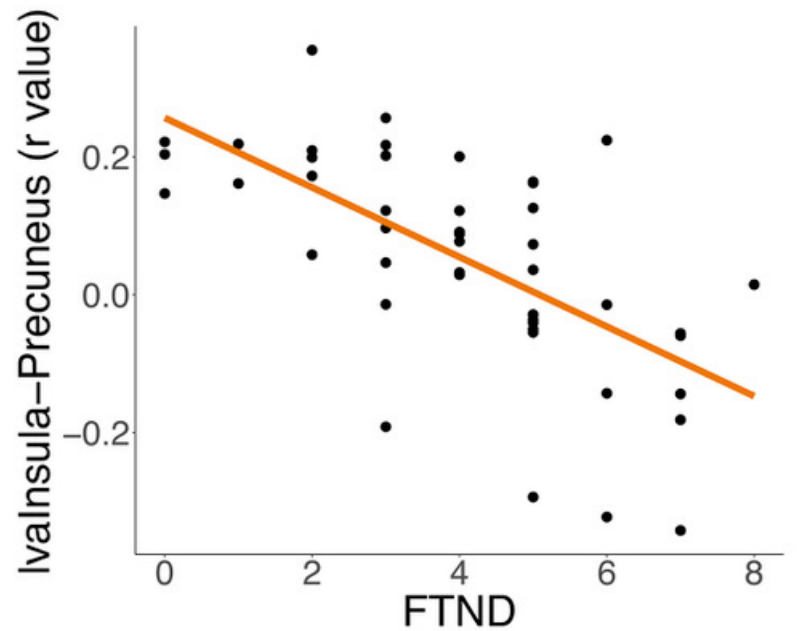

C

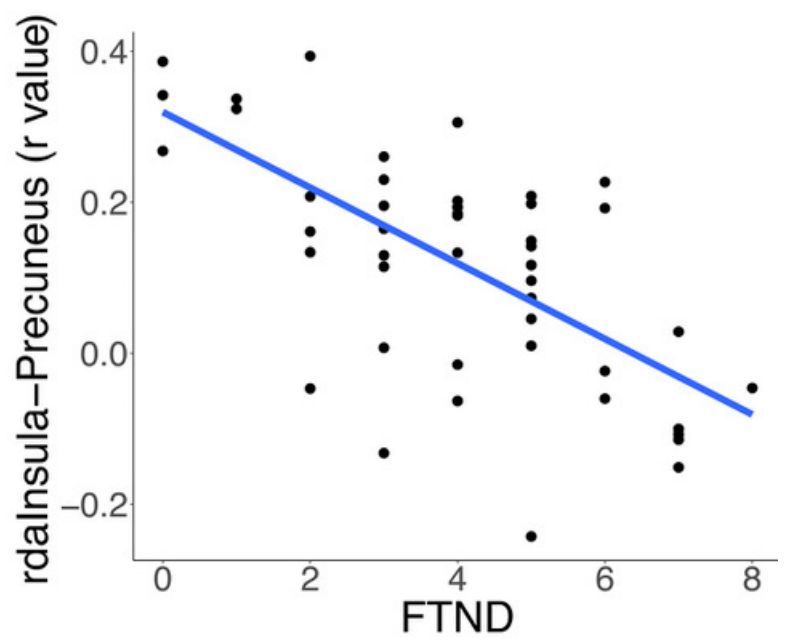


Figure 3

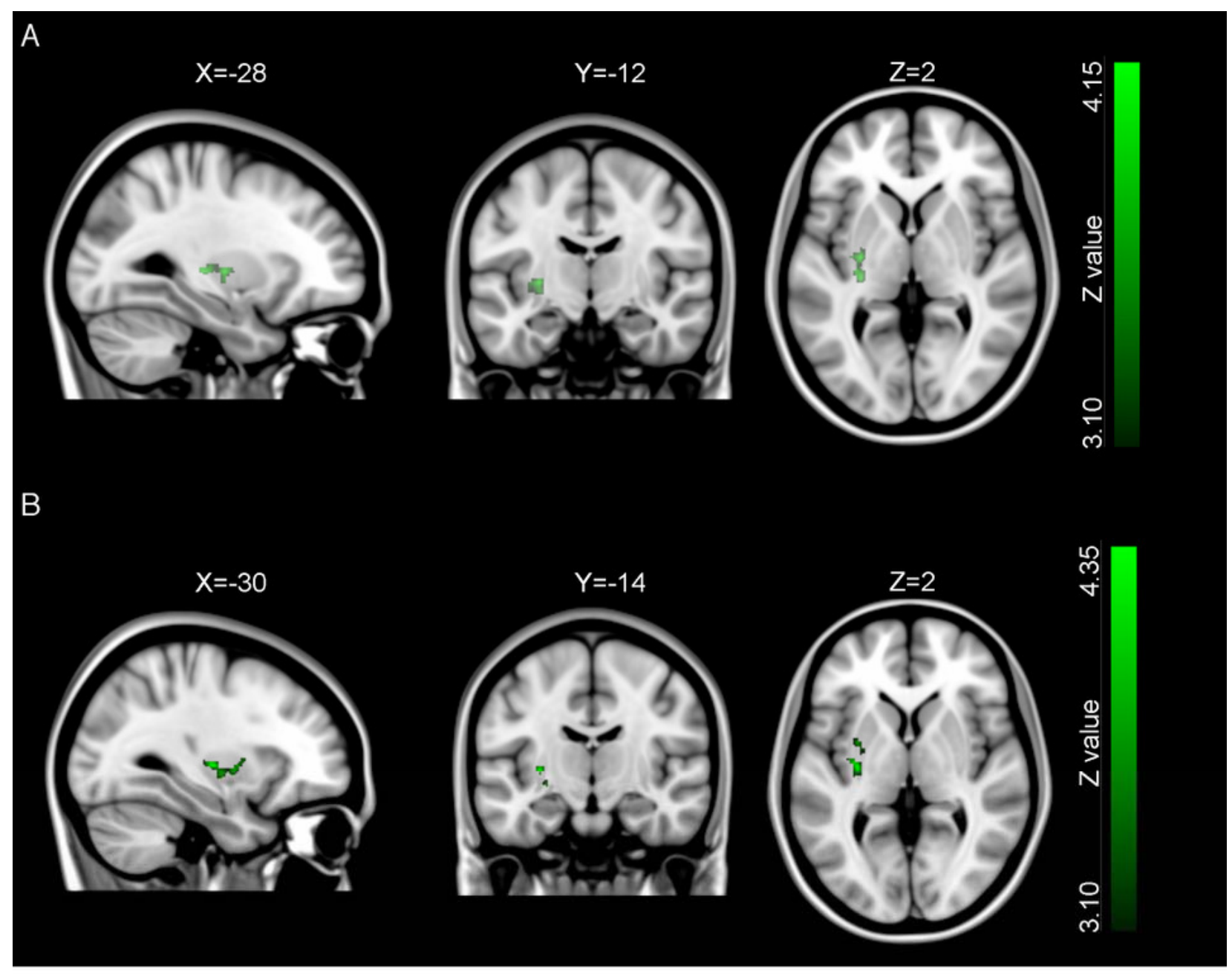

C

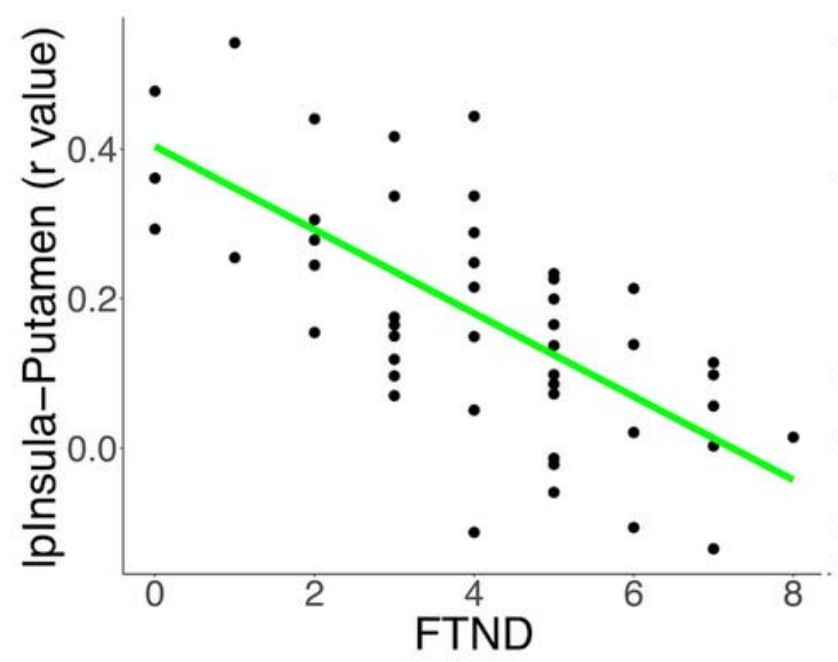

D

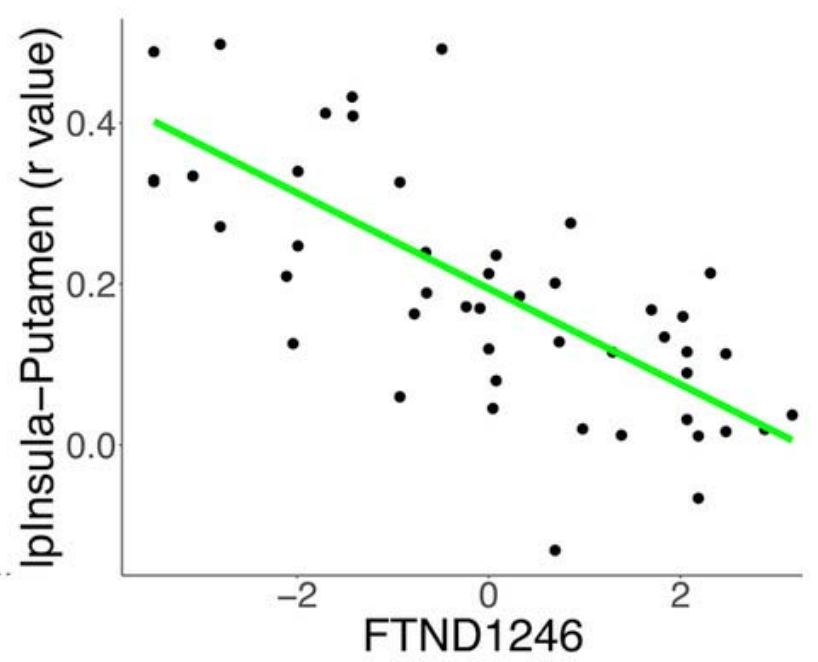

